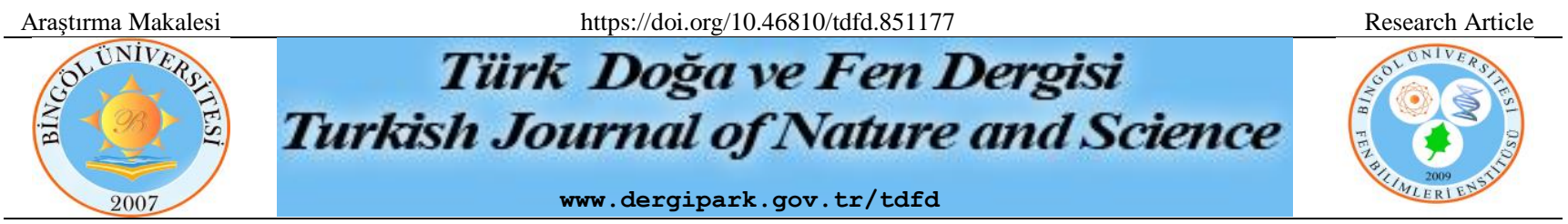

\title{
Hatay’a Özgü Kırmızı Ölmez Çiçek (Helichrysum sanguineum (L.) Kostel ile Sarı Ölmez Çiçeğin (Helichrysum stoeshas spp. barallieri) Biyo Aktif Özellikler
}

\author{
Hasan ASIL ${ }^{1 *}$, Selim TAŞGIN ${ }^{2}$ \\ ${ }^{1}$ Hatay Mustafa Kemal Üniversitesi, Altınözü Tarım Bilimleri MYO, Tıbbi ve Aromatik Bitkiler Programı, Hatay, \\ Türkiye \\ ${ }^{2}$ Zeytincilik Araştırma Enstitüsü, Hatay, Türkiye \\ Hasan ASILL ORCID No: 0000-0002-3690-1789 \\ Selim TAŞGIN ORCID No: 0000-0001-8606-0626 \\ *Sorumlu yazar: hasanasil@mku.edu.tr
}

(Alınış: 31.12.2020, Kabul: 03.06.2021, Online Yayınlanma: 31.12.2021)

\begin{abstract}
Anahtar
Kelimeler

Helichrysum

sanguinem,

Helichrysum

stoeshas spp.

barallieri,

Kirmız1

ölmez çiçek,

Sarı ölmez

çiçek

Öz: Helichrysum türleri genel olarak ölümsüz, ölümsüz çiçek, ölmez çiçeği ve solgun çiçek olarak bilinir ve Anadolu'da çay olarak kullanılmaktadır. Ölmez çiçeğin fenolik bileşikleri koroner kalp hastalığı, inme ve bazı kanser türleri gibi hastalıklara karşı koruma sağladığı ve Ölmez çiçeği uçucu yağları antioksidan maddeler, antiviral, antifungal, antimikrobiyal ve anti-enflamatuar özellikler içermektedir. Bu çalışmada, Helichrysum sanguinem bitkisi ülkemizde sadece Hatay’ın Altınözü ilçesinde bulunmakta olup bu bitkiye uygulanan ultrasonik ekstraksiyon metoduna göre 37 bileşen kayda değer bulunmuştur. Bulunan bileşenler 11 tanesi biyoaktivite özelliği içerirken, 9 tanesi farmakolojik -biyoaktivite ve 3 tane bileşen ise Drog - farmakolojik - biyoaktivite özelliği içermektedir. Helichrysum stoeches spp barellieri bitkisinin ultrasonik ekstraksiyon metoduna göre 19 bileşen kayda değer bulunmuştur. Bulunan bileşenler 2 tanesi biyoaktivite madde içerirken, 5 tanesi farmakolojik ve biyoaktivite madde ve 3 tane bileşen ise Drog - farmakolojik - biyoaktivite özelliği içermektedir.
\end{abstract}

\section{Bio-Active Properties of Hatay-Specific Red Everlasting (Helichrysum sanguineum (L.) Kostel and Yellow Everlasting (Helichrysum stoeshas spp. baraleri)}

\section{Keywords \\ Helichrysum sanguinem, \\ Helichrysum \\ stoeshas spp. \\ barallieri, \\ Red \\ everlasting, \\ Yellow \\ everlasting}

\begin{abstract}
Everlasting, phenolic compounds are used for treatment against diseases such as coronary heart disease, stroke, and some types of cancer. Everlasting essential oils contain antioxidants, antiviral, antifungal, antimicrobial and anti-inflammatory properties. According to the ultrasonic extraction method of Helichrysum sanguinem 37 components were found to be significant. While 11 of them contained bioactivity, 9 of them contained pharmacological and bioactivity, and 3 of them contained Drog - pharmacological - bioactivity. According to the ultrasonic extraction method of Helichrysum stoeches spp barellieri, 19 components were found to be significant. While 2 of them contain bioactivity, 5 of them contain pharmacological and bioactivity agents and 3 of them contain Drog - pharmacological - bioactivity.
\end{abstract}

\section{GİRİş}

Helichrysum Mill. cinsi genel olarak "ölmez çiçek" olarak tanınsa da Anadolu'nun farklı yörelerinde altın otu, sarı çiçek, güneş çiçeği, arı çiçeği, ölümsüz çiçek, solgun çiçek ve yayla çiçeği terimleri de kullanılmaktadır. Tek bir türe özgü yöresel isim alanları da vardır. Bunlar ise "Püren" (H. arenarium), kırmızı guddeme (H. sanguineum), beyaz kurna (H. pamphylicum $)$ ve yoğurt çiçeği $(H$. orientale $)$ gibi yöresel isimler almaktadırlar. Avrupa'da ise "everlasting", "sun gold" (İngiltere), "eternelle", "immortelle" (Fransa), "cmin" (Rusya), "strombloem", "strumblume", "immortelle" (Hollanda), "elicriso", "solfini", "fignamica" (İtalya), "evöighedablomst" (Danimarka), "koçanki" (Polonya), "smill”, "molec", "neven" (Çek Sumhuriyeti) şeklinde isimlendirilmektedir Aslan, [1]; Eroğlu ve ark. [2]; Şen ve Kalayc1, [3]; Eroğlu, [4]. 
Ölmez çiçeği (Helichrysum Mill.) papatyagiller familyasından olup, 600'e yakın türü vardır. Orijini Akdeniz havzası ve Güney Afrika ile Avusturya'dır Ramazanoğlu ve ark. [5]; Baser, [6]; Goldansaz ve ark. [7]. Bu bitkilerin 245'i Güney Afrika'ya özgü, 16's1 Avrupa ve Akdeniz bölgelerinde bulunmaktadır Giovanelli ve ark., [8]. Türkiye'de ise 20 tür ve 26 taksonu bulunmakta ve 15 tanesi endemiktir Davis ve Kupicha, [8]; Kürkçüoğlu ve ark. [6].

Helichrysum sanguineum (L.) Kostel. bitkisinin yaygın kullanılan isimleri, Kırmızı ölmez çiçek, Mesh'in kanı, Kırmızı şarap çiçeği olarak bulandıkları yörelerde adlandırılmaktadır. Coğrafi köken olarak İsrail, Filistin ve Türkiye'de Hatay'ın Altınözü ilçesinde bulunmaktadır. Botanik özellikleri ise yapakları basit, dikdörtgen, mızrak şeklinde, doğrusal, girimsi, yoğun, kurt tüyleri ile örtülüdür. Çiçekleri kırmızı renkli çiçeklerle çevrili yuvarlak sarı çiçeklerdir Giovanelli ve ark., [8]; Ocak ve ark. [10]. Antakya'da çok amaçlı şifalı çay olarak kullanılmaktadır Güzel ve Güzelsemme, [11]. Helichrysum stoechas ssp. barelieri, Sicilya'dan Türkiye'ye geniş bir coğrafyada bulunur ve odunsu, çok yıllık, $10-50 \mathrm{~cm}$ boyunda, çam ormanlarının kenarlarında yetişen ve taşlık yamaçlarda doğal olarak yetişmektedir Doussi ve Thanos, [12].

Helichrysum türleri çok yıllık, otsu veya çalı formunda, yaprakları yoğun ve mızrak şeklindedir. Çiçekleri beyaz veya renkli olup çok sayıda çiçekleri vardır. Küçük sarı çiçekleri kurutulduktan sonra bile altın rengini koruduğundan çiçekçilik sektöründe çok tercih edilmektedir. Avrupa kökenli bir bitki olmasına karşın daha çok Anadolu'da halk tarafindan şifa amaçlı kullanılmıştır. Ölmez çiçek bitkisinden; ölmez çiçek yağı, çayı gibi ürünler üretilir. Ölmez çiçek, idrarın söktürülmesine, böbreklerden kum ve taşların düşürülmesine, sindirim bozukluklarını düzenlenmesine, bağışıklık sisteminin güçlendirilmesine ve vücudun temizlenmesine gibi rahatsızlıklarının tedavisinde 2000 yıldır halk hekimliğinde kullanılmaktadır Ermişler, [13]. Helichrysum'da fenolik bileşikler gibi flavonoidler ve kalkerler, ftalidler, a-piron türevleri, terpenoidler, uçucu yağlar ve yağ asitleri bulunmuştur. Yapılan çalışmalarda fenolik bileşiklerin koroner kalp hastalığı, inme ve bazı kanser türleri gibi hastalıklara karşı koruma sağladığı bildirilmiştir (Eroğlu ve ark. [14]; Goldansaz ve ark. [7].

Balkanlarda ve Türkiye'de yetişen ölmez çiçek türlerinin uçucu yağ bileşenlerinin ana bileşeni pinenlerdir. Ölmez çiçeğinin uçucu yağı, kanayan yaraların tedavisinde kullanılmaktadır. Cilt üzerinde kullanımı ise bir sabit yağda seyreltilerek uygulanmaktadır. Türkiye ise ölmez çiçeği çiçeklerinden elde edilen çay idrar-safra sökücü ve kum dökücü olarak kullanılmaktadır. Ayrıca yapılan çalışmalarda, bu antioksidan bileşiklerin çoğunun, antienflamatuar, anti-aterosklerotik, antitümör, antimutagenik, antikarsinojenik, antibakteriyel ve antiviral aktivitelere sahip olduğunu ve böbrek taşı, ürogenital bozukluklar, mide ağrısı, sarılık, ishal ve astım tedavisinde kullanılmaktadır Aiyegoro ve Okoh, [14].; Eroğlu ve ark. [2]; Boi, [15]; Baser, [6].
$\mathrm{Bu}$ çalışmada, kırmızı ölmez çiçek (Helichrysum sanguineum (L.) Kostel) ve sarı ölmez çiçek (Helichrysum stoechas ssp. barelieri) bitkilerinin ultrasonik ekstraksiyon yöntemi kullanarak GS-MS analizleri yapılarak elde edilen uçucu bileşenler incelenerek bu bitkileri tıbbi özellikleri araştırılmıştır..

\section{MATERYAL VE METOT}

\subsection{Materyal ve Kullanılan Kimyasallar}

Bitki materyalleri Hatay ilinin Altinözü ilçesinde 2018 Mayıs ayında tam çiçeklenme döneminde toplanmış ve aynı gün oda sıcaklığında kurutulmuş ve ışık almayan ortamda muhafaza edilmiştir. Kırmızı ölmez çiçek (Helichrysum sanguineum (L.) Kostel) bitkisi 452 rakımdan ve sarı ölmez çiçek (Helichrysum stoechas ssp. barelieri) bitkisi ise 375 rakımda doğal yetişme alanlarından toplanmıștır. Bitkilerin ekstraksiyonu için gerekli olan çözücülerden Metanol (Isolab, katalog \# 947.043.2500) ve Etil asetat (Tekkim, katalog \# TK.050140.02500) ilgili firmalardan satın alını, alındıkları şekliyle kullanılmıştır.

\subsection{Ekstraksiyon Prosedürü}

Ölmez çiçeği ekstraksiyon işlemi ultrasonik banyo kullanılarak ultrasonik-yardımlı çözücü ekstraksiyonu yöntemine göre gerçekleştirilmiştir (input power $180 \mathrm{~W}$, $35 \mathrm{kHz}$ frekansta ve $25^{\circ} \mathrm{C}$ 'de) (Jalali-Heravi ve ark. [16]; Göktürk ve Asil, [17]. Ölmez çiçeği numunesinin ekstraksiyon işlemi ise şu şekilde yapılmıştır. $1 \mathrm{~g}$ ölmez çiçeği herbası toz haline getirilir. Daha sonra toz halindeki ölmez çiçeği herbası tek boyunlu balona aktarılır ve içerisine $(18-42 \mathrm{~mL})$ metanol:etilasetat (70:30) çözücü karışımı eklenir. Sonikasyon işlemi başlatılır ve 15 dakika karışım sonikasyona tutulur. Sonikasyondan sonra süzgeç kâğıdında süzülür ve sonra $+4{ }^{\circ} \mathrm{C}$ 'de buzdolabında 1 şıksız ortamda GC-MS analizi için bekletilir. GC-MS analizi için bu ekstraktın $1 \mu \mathrm{L}$ 'si kullanııır (Jalali-Heravi ve ark. [16]; Göktürk ve Asil, [17].

\subsection{Gaz Kromatografisi-Kütle Spektroskopisi (GC- MS) Analizi}

GC-MS analizi Hewlett-Packard 6890 serisi GC-MS analiz cihazı kullanılarak gerçekleştirilmiştir. Cihazın kolonu HP-5MS fused silica column (5\% phenyl methyl polysiloxane $30 \mathrm{~m} 0.25 \mathrm{~mm}$ i.d., film thickness $0.25 \mu \mathrm{m}$ ) ve dedektörü Hewlett-Packard mass selective detector 6890'dır. GC-MS analizi literatürde belirtilen prosedüre göre gerçekleştirilmiştir. Fırın $60{ }^{\circ} \mathrm{C}$ 'ye 1 sıtılıp bu sicaklıkta 2 dakika beklenir. Daha sonra sicaklık dakikada 4 derece arttırılarak $180{ }^{\circ} \mathrm{C}$ 'ye, ordan dakikada 2 derece arttırılarak $210{ }^{\circ} \mathrm{C}$ 'ye ve dakikada 5 derece arttırılarak $230{ }^{\circ} \mathrm{C}$ 'ye yükseltilir ve 1 dakika bekletilir. Son olarak sıcaklık dakikada 1 derece artırılarak 280 ${ }^{\circ}$ C'ye yükseltilir Helyum (\%99.9999) taşıyıcı gaz olarak ve $1 \mathrm{~mL} /$ dakika akış hızında kullanılmıştır. Enjektör sicaklığ $1200^{\circ} \mathrm{C}$ 'de tutulmuştur. Ayrılma oranı 1:5'dir. 


\section{BULGULAR}

\subsection{Kırmızı Ölmez Çiçek (Helichrysum sanguinem) ve Sarı Ölmez Çiçek (Helichrysum stoeches spp barellieri) Bitkilerinin Uçucu Bileșenlerinin Karakterizasyonu}

Ölmez çiçek bitkilerinin ultrasonik ekstraksiyon metoduna göre eldilen ucucu bileşenlerden hangilerinin drog, farmakolojik ve biyoaktivite etkiye sahip olduğu literatür (PubChem, Sigma Aldrich ve Pharmacy Research Databases) taramalarıla belirlenmiştir Anonimus, [20,21]. Sarı ölmez çiçek (Helichrysum stoeches spp barellieri) bitkisinin ultrasonik ekstraksiyon metoduna göre bulunan 19 bileșen Tablo 1 'de verilmiştir. Bulunan bileşenler içerisinde en yüksek 11-Octadecenoic acid, methyl ester (\%71.58), 12Octadecenoic acid, methyl ester (\%11.48) ve Heptadecanoic acid, 16-methyl-, methyl ester (\%6.39) bulunmuştur. Bulunan 19 bileşenin 2 tanesi biyoaktivite madde, 5 tanesi farmakolojik ve biyoaktivite, 3 tane bileșen ise drog-farmakolojik- biyoaktivite özellik göstermektedir (Tablo 1).

Kırmızı ölmez çiçek (Helichrysum sanguinem) bitkisinin ultrasonik ekstraksiyon metoduna göre bulunan 37 bileşen Tablo 2'de verilmiştir. Bulunan bileşenler içerisinde en yüksek orana 2-Pyrrolidinone, 1-methyl(\%17.92), Tetradecene, (E)- (\%13.47) ve Butanoic acid, 2-methyl-3-oxo-, methyl ester (\%12.05) bulunmuștur. Bulunan 39 bileşenin 11 tanesi sadece fark biyoaktivite madde içerirken, 9 tanesi farmakolojik ve biyoaktivite, 3 tane bileşen ise drog-farmakolojik- biyoaktivite özellik göstermektedir (Tablo 2).

Öztürk ve ark. [22] Helichrysum plicatum DC. üzerine yaptıkları çalıșmadan Pinene (\%58.4), Hexadecanoic acid (\%15.4), dodecanoic acid (\%9.6) çıkmış bu çalışmada bu kimyasallar düşük çıkmış ancak bu çalışmada 11-Octadecenoic acid, methyl ester (\%71.58), 12-Octadecenoic acid, methyl ester (\%11.48) ve Heptadecanoic acid, 16-methyl-, methyl ester (\%6.39) daha yüksek oranlarda çıkmıştır. Tür ve iklim şartları kimyasal içeriklerin oranlarında değişikliğe neden olmaktadır. Bu bitkilerle ilgili benzer çalışmalar az yapılmış olsa da farklı türünde yapılan bir çalışmada uçucu yağ içeriğine bakılmış ve \% 3.8 oranın da pinene bulmuştur Özkan ve Özcan, [23]. Bu çalışmada sarı ölmez çiçek (Helichrysum stoeches spp barellieri) bitkisinde de pinene bileşeni bulunmuştur.ya resim çözünürlükleri minimum 300 dpi olmalıdır.

Tablo 1. Helichrysum stoeches spp barellieri bitkisinin ultrasonik ekstraksiyon metoduyla elde edilen kimyasal bileşenler

\begin{tabular}{|c|c|c|c|c|c|c|c|}
\hline No & Kimyasal Adı & Kapalı Formül & $\mathrm{M}_{\mathrm{W}}(\mathrm{g} / \mathrm{mol})$ & $\mathrm{tR}$ & $\%$ & MF & Kullanım Alanı \\
\hline 1 & (1R)-(+)-alpha-Pinene & $\mathrm{C}_{10} \mathrm{H}_{16}$ & 136.238 & 6.17 & 0.10 & 91 & Farmakoloji- Biyoaktivite \\
\hline 2 & $\begin{array}{l}\text { Butanoic acid, 2-methyl-3-oxo-, methyl } \\
\text { ester }\end{array}$ & $\mathrm{C}_{6} \mathrm{H}_{10} \mathrm{O}_{3}$ & 130.143 & 8.48 & 0.07 & 9 & Biyoaktivite \\
\hline 3 & 2-Pyrrolidinone, 1-methyl- & $\mathrm{C}_{5} \mathrm{H}_{9} \mathrm{NO}$ & 99.133 & 9.72 & 0.28 & 91 & $\begin{array}{l}\text { Drog - Farmakoloji- } \\
\text { Biyoaktivite }\end{array}$ \\
\hline 4 & L-Fenchone & $\mathrm{C}_{10} \mathrm{H}_{16} \mathrm{O}$ & 152.237 & 10.91 & 0.07 & 64 & Biyoaktivite \\
\hline 5 & 1-Dodecene & $\mathrm{C}_{12} \mathrm{H}_{24}$ & 168.324 & 14.27 & 0.19 & 72 & Farmakoloji- Biyoaktivite \\
\hline 6 & 1-Tridecene & $\mathrm{C}_{13} \mathrm{H}_{26}$ & 182.351 & 20.86 & 0.34 & 91 & Biyoaktivite \\
\hline 7 & Butylated Hydroxytoluene & $\mathrm{C}_{15} \mathrm{H}_{24} \mathrm{O}$ & 220.356 & 24.66 & 0.07 & 72 & $\begin{array}{c}\text { Drog - Farmakoloji- } \\
\text { Biyoaktivite }\end{array}$ \\
\hline 8 & 1-Hexadecanol & $\mathrm{C}_{16} \mathrm{H}_{34} \mathrm{O}$ & 242.447 & 26.88 & 0.33 & 91 & $\begin{array}{l}\text { Drog - Farmakoloji- } \\
\text { Biyoaktivite }\end{array}$ \\
\hline 9 & 2-Dodecanol & $\mathrm{C}_{12} \mathrm{H}_{26} \mathrm{O}$ & 186.339 & 32.33 & 0.17 & 80 & Biyoaktivite \\
\hline 10 & Hexadecanoic acid, methyl ester & $\mathrm{C}_{17} \mathrm{H}_{34} \mathrm{O}_{2}$ & 270.457 & 36.78 & 0.20 & 96 & Farmakoloji- Biyoaktivite \\
\hline 11 & $\begin{array}{l}\text { 9,12-Octadecadienoic acid (Z,Z)-, methyl } \\
\text { ester }\end{array}$ & $\mathrm{C}_{19} \mathrm{H}_{34} \mathrm{O}_{2}$ & 294.479 & 46.16 & 1.84 & 98 & Farmakoloji- Biyoaktivite \\
\hline 12 & $\begin{array}{l}\text { 9,12-Octadecadienoic acid (Z,Z)-, methyl } \\
\text { ester }\end{array}$ & $\mathrm{C}_{19} \mathrm{H}_{34} \mathrm{O}_{2}$ & 294.479 & 46.29 & 1.84 & 98 & Farmakoloji- Biyoaktivite \\
\hline 13 & 11-Octadecenoic acid, methyl ester & $\mathrm{C}_{19} \mathrm{H}_{36} \mathrm{O}_{2}$ & 296.495 & 47.04 & 71.58 & 99 & Bulunamad1 \\
\hline 14 & 12-Octadecenoic acid, methyl ester & $\mathrm{C}_{19} \mathrm{H}_{36} \mathrm{O}_{2}$ & 296.495 & 47.22 & 11.48 & 99 & Bulunamad1 \\
\hline 15 & 9-Octadecenoic acid, methyl ester & $\mathrm{C}_{19} \mathrm{H}_{36} \mathrm{O}_{2}$ & 296.495 & 47.58 & 4.96 & 99 & Biyoaktivite \\
\hline 16 & Methyl isostearate & $\mathrm{C}_{19} \mathrm{H}_{38} \mathrm{O}_{2}$ & 298.511 & 48.73 & 6.39 & 98 & Bulunamadı \\
\hline 17 & 10-Octadecenoic acid, methyl ester & $\mathrm{C}_{19} \mathrm{H}_{36} \mathrm{O}_{3}$ & 312.5 & 58.28 & 0.39 & 91 & Bulunamadı \\
\hline 18 & 15-Octadecenoic acid, methyl ester & $\mathrm{C}_{19} \mathrm{H}_{36} \mathrm{O} 2$ & 296.5 & 58.35 & 0.04 & 91 & Bulunamad1 \\
\hline 19 & Eicosanoic acid, methyl ester & $\mathrm{C}_{21} \mathrm{H}_{42} \mathrm{O}_{2}$ & 326.6 & 60.93 & 0.38 & 86 & Bulunamad1 \\
\hline
\end{tabular}

Tablo 2. Helichrysum sanguineum $(L$, ) Kostel bitkisinin ultrasonik ekstraksiyon metoduyla elde edilen kimyasal bileșenler

\begin{tabular}{|c|c|c|c|c|c|c|c|}
\hline No & Kimyasal Adı & Kapalı Formül & $\begin{array}{c}\mathrm{M}_{\mathrm{W}} \\
(\mathrm{g} / \mathrm{mol})\end{array}$ & $\mathrm{tR}$ & $\%$ & MF & Kullanım Alanı \\
\hline 1 & 1,6-Heptadien-3-yne, 5-methyl- & $\mathrm{C}_{8} \mathrm{H}_{10}$ & 109.16 & 5.22 & 0.63 & 4 & Bulunamadı \\
\hline 2 & Butanoic acid, 2-methyl-3-oxo-, methyl ester & $\mathrm{C}_{6} \mathrm{H}_{10} \mathrm{O}_{3}$ & 130.143 & 5.89 & 12.05 & 78 & Biyoaktivite \\
\hline 3 & 3-Acetoxybenzoic acid & $\mathrm{C}_{9} \mathrm{H}_{8} \mathrm{C}_{4}$ & 180.16 & 6.17 & 3.06 & 1 & Bulunamadi \\
\hline 4 & Furan, 2-methoxy- & $\mathrm{C}_{5} \mathrm{H}_{6} \mathrm{O}_{2}$ & 98.101 & 6.53 & 0.91 & 9 & Biyoaktivite \\
\hline 5 & 2-Norbornene & $\mathrm{C}_{7} \mathrm{H}_{10}$ & 94.157 & 7.37 & 2.15 & 3 & Biyoaktivite \\
\hline 6 & Methyl vinyl ketone & $\mathrm{C}_{7} \mathrm{H}_{10}$ & 70.091 & 7.71 & 0.72 & 4 & $\begin{array}{l}\text { Farmakoloji } \\
\text { Biyoaktivite }\end{array}$ \\
\hline 7 & Methyl propionate & $\mathrm{C}_{4} \mathrm{H}_{8} \mathrm{O}_{2}$ & 88.106 & 8.48 & 1.35 & 4 & $\begin{array}{l}\text { Farmakoloji } \\
\text { Biyoaktivite }\end{array}$ \\
\hline 8 & Acetamide, 2-fluoro & $\mathrm{C}_{2} \mathrm{H}_{4} \mathrm{FNO}$ & 77.058 & 8.94 & 0.96 & 2 & Farmakoloji \\
\hline
\end{tabular}




\begin{tabular}{|c|c|c|c|c|c|c|c|}
\hline & & & & & & & Biyoaktivite \\
\hline 9 & 2-Pyrrolidinone, 1-methyl- & $\mathrm{C}_{5} \mathrm{H}_{9} \mathrm{NO}$ & 99.133 & 9.65 & 17.92 & 91 & $\begin{array}{c}\text { Drog } \\
\text { Farmakoloji } \\
\text { Biyoaktivite }\end{array}$ \\
\hline 10 & 3-Furanmethanol & $\mathrm{C}_{5} \mathrm{H}_{6} \mathrm{O}_{2}$ & 98.101 & 9.97 & 0.33 & 4 & Biyoaktivite \\
\hline 11 & 2-Furanmethanol & $\mathrm{C}_{5} \mathrm{H}_{6} \mathrm{O}_{2}$ & 98.101 & 10.02 & 0.26 & 5 & $\begin{array}{l}\text { Farmakoloji } \\
\text { Biyoaktivite }\end{array}$ \\
\hline 12 & Methyl benzoate & $\mathrm{C}_{8} \mathrm{H}_{8} \mathrm{O}_{2}$ & 136.15 & 11.13 & 2.73 & 40 & $\begin{array}{l}\text { Farmakoloji } \\
\text { Biyoaktivite }\end{array}$ \\
\hline 13 & Cyclododecane & $\mathrm{C}_{12} \mathrm{H}_{24}$ & 168.324 & 14.29 & 7.19 & 96 & Biyoaktivite \\
\hline 14 & Ethane, 1-chloro-2-isocyanato- & $\mathrm{C}_{3} \mathrm{H}_{4} \mathrm{CINO}$ & 105.521 & 17.97 & 0.23 & 3 & Biyoaktivite \\
\hline 15 & 2-Tetradecene, $(\mathrm{E})$ - & $\mathrm{C}_{14} \mathrm{H}_{28}$ & 196.378 & 20.90 & 13.47 & 97 & Bulunamadı \\
\hline 16 & 1-Propene, 3-azido- & $\mathrm{C}_{3} \mathrm{H}_{5} \mathrm{~N}_{3}$ & 83.09 & 21.05 & 0.24 & 3 & Bulunamadı \\
\hline 17 & Pyridine & $\mathrm{C}_{5} \mathrm{H}_{5} \mathrm{~N}$ & 79.102 & 21.85 & 0.62 & 2 & $\begin{array}{l}\text { Farmakoloji } \\
\text { Biyoaktivite }\end{array}$ \\
\hline 18 & 2-Ethylidenehydrazono-3-methyl-2,3 & $\mathrm{C}_{10} \mathrm{H}_{11} \mathrm{~N}_{3} \mathrm{~S}$ & 205.28 & 24.72 & 0.35 & 2 & Bulunamad \\
\hline 19 & 5-Octadecene, $(\mathrm{E})-$ & $\mathrm{C}_{18} \mathrm{H}_{36}$ & 252.486 & 26.91 & 10.12 & 91 & Bulunamad1 \\
\hline 20 & Methane, isocyanato- & $\mathrm{CH}_{3} \mathrm{NCO}$ & 57.052 & 27.07 & 0.22 & 1 & $\begin{array}{l}\text { Farmakoloji } \\
\text { Biyoaktivite }\end{array}$ \\
\hline 21 & 2-Dodecanol & $\mathrm{C}_{12} \mathrm{H}_{26} \mathrm{O}$ & 186.339 & 32.34 & 4.75 & 72 & Biyoaktivite \\
\hline 22 & Cyclohexylmethyl formate & $\mathrm{C}_{8} \mathrm{H}_{14} \mathrm{O}_{2}$ & 142.198 & 46.58 & 2.40 & 10 & Bulunamad1 \\
\hline 23 & Cyclopropane, 1-ethyl-1-methyl- & $\mathrm{C}_{6} \mathrm{H}_{12}$ & 84.16 & 46.62 & 0.85 & 25 & Bulunamadı \\
\hline 24 & 1-Butanamine, 3-methyl- & $\mathrm{C}_{5} \mathrm{H}_{13} \mathrm{~N}$ & 84.166 & 60.88 & 0.14 & 3 & $\begin{array}{c}\text { Drog } \\
\text { Farmakoloji } \\
\text { Biyoaktivite }\end{array}$ \\
\hline 25 & 3-Pyrrolidinol & $\mathrm{C}_{4} \mathrm{H}_{9} \mathrm{NO}$ & 87.122 & 60.95 & 0.52 & 4 & Biyoaktivite \\
\hline 26 & 1-Propanol, 2-methyl- & $\mathrm{C}_{4} \mathrm{H}_{10} \mathrm{O}$ & 74.123 & 61.01 & 0.44 & 3 & $\begin{array}{c}\text { Drog } \\
\text { Farmakoloji } \\
\text { Biyoaktivite }\end{array}$ \\
\hline 27 & Indolizine, 2-(4-methylphenyl)- & $\mathrm{C}_{15} \mathrm{H}_{13} \mathrm{~N}$ & 207.276 & 79.13 & 2.15 & 2 & Biyoaktivite \\
\hline 28 & 6-Nitro-8-methoxy-2H-chromene & $\mathrm{C}_{10} \mathrm{H}_{9} \mathrm{NO}_{4}$ & 207.18 & 72.25 & 1.22 & 2 & Bulunamadı \\
\hline 29 & Methane, isocyanato- & $\mathrm{CH}_{3} \mathrm{NCO}$ & 57.052 & 79.35 & 0.16 & 1 & $\begin{array}{l}\text { Farmakoloji } \\
\text { Biyoaktivite }\end{array}$ \\
\hline 30 & $\begin{array}{l}\text { Cyclohexane-1,3-dione, 2-allylaminomethylene-5,5- } \\
\text { dimethyl- }\end{array}$ & $\mathrm{C}_{12} \mathrm{H}_{17} \mathrm{NO}_{2}$ & 207.273 & 79.81 & 1.04 & 2 & Biyoaktivite \\
\hline 31 & 2-Hydroxyhexadecanoic acid & $\mathrm{C}_{16} \mathrm{H}_{32} \mathrm{O}_{3}$ & 272.42 & 81.08 & 1.42 & 4 & $\begin{array}{l}\text { Farmakoloji } \\
\text { Biyoaktivite }\end{array}$ \\
\hline 32 & 4-Dodecanol & $\mathrm{C}_{12} \mathrm{H}_{26} \mathrm{O}$ & 186.339 & 81.89 & 0.50 & 2 & Biyoaktivite \\
\hline 33 & 2,4-Dimethylbenzo[h]quinoline & $\mathrm{C}_{15} \mathrm{H}_{13} \mathrm{~N}$ & 207.27 & 82.38 & 1.04 & 2 & Bulunamad1 \\
\hline 34 & $\begin{array}{l}\text { 2-Chloro-4,6-bis[3-(perfluorohexyl)propyloxy]-1,3,5- } \\
\text { triazine }\end{array}$ & $\mathrm{C}_{21} \mathrm{H}_{12} \mathrm{ClF}_{26} \mathrm{~N}_{3} \mathrm{O}_{2}$ & 867.7 & 82.74 & 0.72 & 4 & Bulunamad \\
\hline 35 & 1,4-Di[3-(3-isothiocyanatophenyl)thioureido]butane & $\mathrm{C}_{20} \mathrm{H}_{20} \mathrm{~N}_{6} \mathrm{~S}_{4}$ & 472.7 & 83.09 & 0.61 & 2 & Bulunamadı \\
\hline 36 & Formic acid & $\mathrm{CH}_{2} \mathrm{O}_{2}$ & 46.025 & 83.53 & 0.24 & 2 & Bulunamad1 \\
\hline 37 & 2-1-Phenyl ethylidene-hydrazono-3- & $\mathrm{C}_{20} \mathrm{H}_{21} \mathrm{~N}_{3} \mathrm{OS}$ & 351.5 & 83.66 & 4.73 & 3 & Bulunamad1 \\
\hline
\end{tabular}

\section{SONUÇ}

$\mathrm{Bu}$ çalışmanın sonuçları; Helichrysum sanguineum (L,) Kostel ve Helichrysum stoeches spp barellieri bitkilerinin metanol/etil asetat karışımı ile elde edilen ekstraktlarında birçok biyoaktif bileşen bulunmuştur. Bu biyoaktif bileşenler biyoaktivite, drog ve farmakolojik aktivite içermektedir. Bu çalışmada elde edilen sonuçlar ile kırmızı ölmez çiçek (Helichrysum sanguineum (L,) Kostel) ve sarı ölmez çiçek (Helichrysum stoeches spp barellieri) bitkilerinin tıbbi bitki olma özelliğini göstermektedir. $\mathrm{Bu}$ çalışma bu nedenle ileriki tıbbi bitkilerle yapılacak çalışmalar için örnek bir çalışma olup, bu aktif bileşenlerin izolasyonunu ve saflaştırılıp ilaç sanayinde kullanılmasının sağlanması hususunda yararlı olacağı kanaatindeyiz.

\section{KAYNAKLAR}

[1] Aslan, M, Helichrysum licatum ssp, plicatum Üzerinde Farmakognozik Araştırmalar, Gazi Üniversitesi Sağllk Bilimleri Enstitüsü, Farmakognozi Anabilim Dalı, Yüksek Lisan tezi, 76 sayfa, 1994.
[2] Eroğlu EH, Hamzaoğlu E, Aksoy A, Budak Ü, Özkul Y,. In Vitro Genotoxic Effects of Four Helichrysum Species in Human Lymphocytes Cultures,, Biological Research 43 (2):177-183, 2010.

[3] Şen N, Kalaycı G, Altın Otu Bitkisinden (Helichrysum arenarium) Tanen ve Kumarinin Kimyasal Kompozisyonu, Selçuk Üniversitesi Fen Fakültesi Fen Dergisi, 42(2), 226-231, 2016.

[4] Eroğlu H. The Names in Turkish and Other Languages of Turkey Helichrysum Taxa, Avrasya Terim Dergisi, 6 (1), 26-34, Retrieved from http://dergipark,gov,tr/ejatd/issue/37229/4296. 2018

[5] Ramazanoğlu N, Açık L, Aslan S, Vural M. Antioxidant Activities of Endemic Helichrysum chionophilum, 6th Conference on Medicinal and Aromatic Plants of Southeast European Countries (CMAPSEEC) Proceedings, 2014.

[6] Kürkçüoğlu M, Agalar HG, Aksoy A, Başer KHC. Composition of the Essential Oils of Two Endemic Helichrysum Species in Turkey, 2019.

[7] Goldansaz SM, Mahboubi A, Yazdi-nejad A, Jahanbakhshi M, Mojab F.Investigation on total phenolic content, antibacterial, and antioxidant activity of ethanolic extract of Helichrysum 
leucocephalum Boiss, American Journal of Essential Oils and Natural Products, , 1, 2018.

[8] Giovanelli S, De Leo M, Cervelli C, Ruffoni B, Ciccarelli D, et al.Essential Oil Composition and Volatile Profile of Seven Helichrysum Species Grown in Italy, Chemistry \& biodiversity, 15(5), e1700545, 2018.

[9] Davis PH, Kupicha FK. Helichrysum, -In: Davis, P, $\mathrm{H}$, (ed,), Flora of Turkey and the East Aegean Islands, 5: 80-97, Edinburgh University Press, Edinburgh, 1975.

[10] Ocak A, Kayıkçı S, Güzel Y, Antakya'nın Doğal Bitkileri, Hatay Büyükșehir Belediyesi Kültür Yayınlar1 No:3 113, 2014.

[11] Guzel Y, Guzelsemme M. Wild plants used as herbal tea in Antakya and Defne provinces of Hatay, Anadolu, 28(1), 1-5, 2018.

[12] Doussi MA, Thanos CA. Ecophysiology of seed germination in composites inhabiting fire-prone Mediterranean ecosystems, In Basic and applied aspects of seed biology (pp, 641-649), Springer, Dordrecht, 1997.

[13] Ermişler A. Altın Otu (Helıchrysum Arenarıum) ve Fesleğen (Ocımum Basılıcum) Bitkilerinin Sinek Kovucu(Repellent) Özelliklerinin Karşılaştırılması, Selçuk Üniversitesi Fen Bilimleri Enstitüsü Kimya Anabilim Dalı Yüksek Lisans Tezi, 65, 2017.

[14] Aiyegoro OA, Okoh AI. Preliminary phytochemical screening and in vitro antioxidant activities of the aqueous extract of Helichrysum longifolium DC, BMC Complementary and Alternative medicine, 10(1), 21, 2010.

[15] Boi M. The Ethnocultural significance for the use of plants in Ancient Funerary Rituals and its possible implications with pollens found on the Shroud of Turin, Ben Congreso Internacional sobre la Sábana Santa en España,, 15, 2012.

[16] Jalali-Heravi M, Parastar H, Ebrahimi-Najafabadi H. Characterization of volatile components of Iranian saffron using factorial-based response surface modeling of ultrasonic extraction combined with gas chromatography-mass spectrometry analysis, Journal of Chromatography A, 1216: 6088-6097, 2009.

[17] Göktürk E, Asil H, Hatay/Kırıkhan'da Yetiştirilen Safran (Crocus sativus L,) Stigmasının Ekstraktının GC-MS analizi, Türk Tarım ve Doğa Bilimleri Dergisi, 5 (3), 317-321, DOI: 10,30910/turkjans,448375, 2018.

[18] Arpag OF, Duran N, Açikgül FC, Türkmen M.. Comparison of Minimum Inhibitory Concentrations of Hypericum Perforatum L. Essential Oils, 0.2\% Chlorhexidine and $10 \%$ Povidone-iodine Over Aggregatibacter actinomycetemcomitans and Porphyromonas gingivalis. Journal of Essential Oil Bearing Plants, 23(6), 1192-1205, 2020.

[19] Türkmen M, Mert A. Farklı azot dozlarının kişniş (Coriandrum sativum L.) yaş herba uçucu yağ bileşenleri üzerine etkisi. Mustafa Kemal Üniversitesi Tarım Bilimleri Dergisi, 25(3), 309$315,2020$.

[20] Anonimus 2018c, https://pubchem.ncbi.nlm.nih.gov/search/
[21] Anonimus 2018d, https://www.sigmaaldrich.com/chemistry.html

[22] Öztürk B, Özek G, Özek T, Başer KHC. Chemical Diversity in Volatiles of Helichrysum, 2014.

[23] Özkan G, Özcan M. Gc/Ms Study Of Essentıal O1l From Helichrysum Chasmolycicum Ph Davis, Selçuk Tarım Bilimleri Dergisi, 20(40), 9-11, 2006. 